\title{
A constructive debate
}

\author{
Synthetic chemistry has long been used to prepare useful compounds - especially those that are hard to obtain from \\ natural sources. But synthetic biology is coming of age as an alternative strategy. A biologist and two chemists debate the \\ merits of their fields' synthetic prowess.
}

\section{Building with biology}

\section{JAY D. KEASLING}

Cynthetic biology is essentially the assembly of well-characterized biological components into a system that performs a function, such as synthesizing a chemical. The field has advanced to the point that one can imagine producing nearly any organic molecule even those that are not produced naturally - in an engineered microorganism. This has enormous implications for the production of speciality and bulk chemicals, drugs and fuels.

Structurally complex pharmaceutical ingredients based on natural products are particularly good targets for microbial production (Fig. 1a), because they can be difficult to produce by conventional chemical synthesis. Even when chemical syntheses for natural products are available, the routes used are often too long and/or low-yielding for large-scale preparation. For commercial production, such molecules are therefore typically harvested from organisms that produce them naturally, or from a mutant that generates higher yields. Alternatively, a semi-synthesis can be used in which a precursor to a desired compound is obtained from an organism and then converted to the final product using organic synthesis. However, these approaches tend to be time-consuming and expensive.

Naturally occurring compounds can be produced in microorganisms by transferring product-specific enzymes, or even whole metabolic pathways, from rare and/or genetically intractable organisms to those that can be readily engineered ${ }^{1}$. Similarly, fuels, bulk chemicals and speciality chemicals that are not produced naturally can be obtained by combining enzymes or metabolic pathways from different hosts into a single microorganism, or by engineering enzyme functions ${ }^{2}$.

Synthetic biology has also been used for the large-scale semi-synthesis of natural products. For example, the antimalarial drug artemisinin is extracted from the plant Artemisia annua,
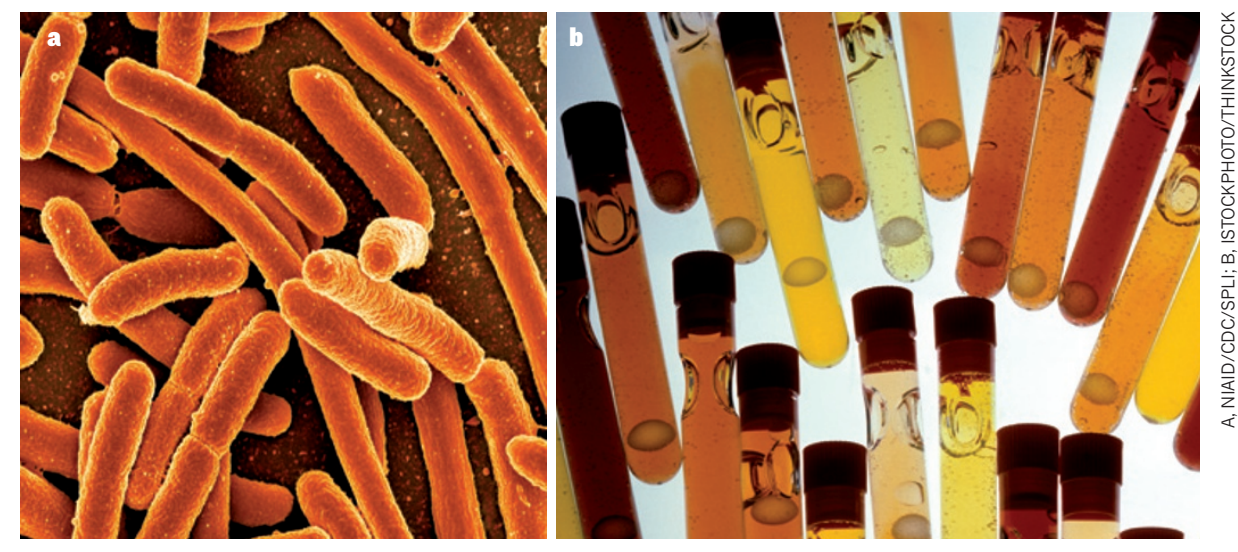

Figure 1 | Reaction vessels. a, Some biologists have argued that structurally complex molecules are best prepared in genetically engineered organisms, such as the bacterium Escherichia coli (pictured). b, Others think that chemical methods will endure as the most general option for synthesizing any desired compound.

but it is in short supply and is too expensive for most people with malaria ${ }^{3}$. By combining genes from A. annua and other organisms into a single strain of the yeast Saccharomyces cerevisiae, we have produced a fermentation process ${ }^{4}$ to make artemisinic acid - which can easily be converted to artemisinin using chemical methods ${ }^{5}$ - from simple sugars. The process is cost-effective, environmentally friendly and reliable, and is being developed for commercial production of the drug.

Synthetic biology has many advantages over chemical synthesis. First, the intermediates in a biosynthesis do not need to be purified before being used as substrates in the next reaction. Second, the many 'protection' and 'de-protection' steps typical of chemical synthesis - steps in which chemical groups are temporarily modified to stop them taking part in unwanted side reactions - can be avoided, because biosynthetic enzymes catalyse reactions only at the required positions in a substrate, avoiding side reactions at other groups. Third, most products of enzymatic reactions are racemically pure (the products form as just one of two possible mirror-image isomers), which is important for biologically active molecules. Fourth, cells can be engineered to secrete the final product, making it easier to purify. Finally, synthetic biology can use simple starting materials from renewable sources, helping to reduce our dependence on oil-derived feedstocks.

Even so, if synthetic biology is to match the power of synthetic chemistry, several problems must be addressed. For example, the biosynthetic enzymes responsible for producing important natural products need to be identified, so that they can be used in syntheses. We must also improve our ability to design enzymes that catalyse reactions not found in nature ${ }^{6}$ if we are to expand the types of chemistry that can be engineered into cells. And we must learn how to reliably engineer biosynthetic pathways to achieve desired outcomes.

I envisage a day when customized cells will be built as catalysts for the biosynthesis of natural products, by designing chromosomes that harbour genes encoding the necessary biosynthetic pathways and also the minimal set of genes needed to construct the host organism from minimal nutrients. That day is fast approaching.

Jay D. Keasling is in the Department of Chemical and Biomolecular Engineering, and the Department of Bioengineering, University of California, Berkeley, Berkeley, California 94720, USA. He is also at the Lawrence Berkeley National Laboratory and at the Joint BioEnergy Institute in California. e-mail: keasling@berkeley.edu 


\section{Practical chemistry}

\section{ABRAHAM MENDOZA \& PHIL S. BARAN}

ince the birth of synthetic chemistry about 180 years ago, society has loved the wonders bestowed by the field, such as life-saving medicines, pest control and molecules that light up telephone displays, yet has harshly criticized it as being solely responsible for pollution and environmental harm. Synthetic biology emerged as an alternative for constructing molecules only about ten years ago, but some have already proclaimed ${ }^{7}$ that it will supplant chemical synthesis. No one should doubt the usefulness of synthetic biology, or its potential to shorten synthetic routes and reduce waste in chemical production. But we are convinced that synthetic chemistry will continue to dominate for the foreseeable future (Fig. 1b), for three main reasons.

The first reason is that chemical synthesis is the best way to solve supply problems. For decades, synthetic chemistry has provided sufficient quantities of agrochemicals, medicines, perfumes and materials for society's needs. The pharmaceutical industry in particular relies on chemical methods for the large-scale production of most small-molecule drugs. The majority of these compounds are based on molecular structures not found in nature, which means that they cannot be prepared through enzymatic processes and are likely to be toxic to the host organisms used in synthetic biology. Synthetic biology has had a crucial impact on the commercial production of some medicines derived from complex natural products, such as artemisinin ${ }^{2}$ and the anticancer drug paclitaxel (Taxol $)^{8}$. But natural products are essentially the only compounds for which biological syntheses can compete with chemical ones, because evolution has optimized the biosynthesis of those products over time.

So the supply of chemicals is best addressed by synthetic chemistry, unless a specific natural product is required in large quantities - and even then, semi-synthetic strategies involving a few chemical steps are often required. In fact, total chemical syntheses of natural products are becoming increasingly efficient and scalable, as demonstrated by the impressive routes used to make tetracycline antibiotics ${ }^{9}$ and the anticancer agent eribulin ${ }^{10}$. A practical chemical synthesis of artemisinin has also now emerged ${ }^{11}$ that could form the basis of an industrial-scale process for making the drug, and a large-scale synthesis of Taxol is being developed ${ }^{12}$.

Optimizing the properties of useful compounds, or adapting their functions to new applications, often requires modification of their molecular structures. The second reason that synthetic chemistry will endure is that chemical methods provide a reliable set of tools to do this in many fundamentally different cases. Moreover, unlike biological syntheses, chemical syntheses can often be developed and implemented rapidly, which is a great advantage.

The third reason is that chemistry excels in the invention of unnatural molecules that have desirable physical properties - such as dyes for printable organic solar cells, fluorescent probes for biological research or radiolabelled drugs used in medicine. The molecular needs of vibrant modern fields such as supramolecular chemistry, chemical biology and nanotechnology can be addressed only by synthetic chemistry. This is partly because the required molecules contain motifs that nature cannot assemble or that would be toxic to host organisms when biosynthesized at the concentrations required for a practical production process. The exponential pace of development of these fields also means that the compounds needed are continuously changing, limiting the time available to synthesize and evaluate them. General chemical methods that can be applied quickly are therefore much more suitable for making such compounds than bioengineering.

Over the years, many people have advocated alternatives to synthetic chemistry or expressed the opinion that it is already a mature field and that all future advances will be incremental at best. But the field is as lively as ever: a seemingly infinite number of problems are waiting to be solved, and legions of talented students are eager to solve them. Synthetic biology surely has a bright future, but no approach to making molecules is more generally useful and has such seemingly limitless potential than synthetic chemistry. It is here to stay.

Abraham Mendoza and Phil S. Baran are in the Department of Chemistry, The Scripps Research Institute, La Jolla, California 92037, USA.

e-mail:pbaran@scripps.edu

1. Malpartida, F. \& Hopwood, D. A. Nature 309 462-464 (1984).

2. Steen, E. J. et al. Nature $463,559-562$ (2010).

3. Enserink, M. Science 307, 33 (2005).

4. Ro, D. K. et al. Nature 440, 940-943 (2006)

5. Acton, N. \& Roth, R. J. J. Org. Chem. 57, 3610-3614 (1992).

6. Siegel, J. B. et al. Science 329, 309-313 (2010)

7. McDaniel, R. \& Weiss, R. Curr. Opin. Biotechnol. 16, 476-483 (2005).

8. Bringi, V., Kadkade, P. G., Prince, C. L. \& Roach, B. L. US patent 7264951 (2007)

9. Charest, M. G., Lerner, C. D., Brubaker, J. D., Siegel, D. R. \& Myers, A. G. Science 308, 395-398 (2005).

10.Ledford, H. Nature 468, 608-609 (2010)

11.Zhu, C. \& Cook, S. P. J. Am. Chem. Soc. 134, 13577-13579 (2012).

12. Mendoza, A., Ishihara, Y. \& Baran, P. S. Nature Chem. 4, 21-25 (2012).

\title{
CELL BIOLOGY
}

\section{All clear for ribosome landing}

\begin{abstract}
The discovery of a dramatic structural rearrangement that is stabilized by an RNA scaffold helps to explain how nascent proteins are delivered for export from the cell cytoplasm. SEE LETTER P.271
\end{abstract}

\section{HARRIS D. BERNSTEIN}

$\mathrm{P}$ rotein sorting within a cell is not an easy undertaking. The synthesis of many proteins destined to leave the cell or to become part of a cellular membrane must be coupled with their transport across or into the appropriate membrane. A universal RNAprotein complex called the signal recognition particle (SRP) plays an essential role in this process by interacting with ribosomes, the cell's protein-synthesizing factories, while they are engaged in protein translation. SRP then escorts the translating ribosomes to a cellular organelle known as the endoplasmic reticulum or, in bacteria, to the cytoplasmic membrane, where they dock onto a transport channel called the Sec complex, or SecYEG in bacteria. SRP must presumably be displaced from the ribosome for docking to happen, but the mechanism of this molecular exchange has been an enigma. On page 271 of this issue, Shen et al. ${ }^{1}$ provide compelling evidence that a remarkable conformational change driven by the RNA component of SRP enables the ribosome to deliver its protein cargo efficiently.

Most membrane and secreted proteins that emerge from translating ribosomes carry a signal peptide - a sequence at their amino terminus that earmarks them for export from the cytoplasm. SRP recognizes and binds to this peptide and then interacts with a membrane receptor (FtsY) before releasing its nascentprotein cargo and transferring the ribosome to SecYEG (Fig. 1). This co-translational mode of protein targeting ensures that proteins destined to leave the cytoplasm begin their journey before they aggregate or fold into a structure that cannot fit through the SecYEG channel.

Although SRP has undergone dramatic 